\title{
Investigation of High-Frequency Internal Wave Interactions with an Enveloped Inertia Wave
}

\author{
B. Casaday ${ }^{1}$ and J. Crockett ${ }^{2}$ \\ ${ }^{1}$ Department of Mechanical Engineering, Ohio State University, Columbus, OH 43210, USA \\ ${ }^{2}$ Department of Mechanical Engineering, Brigham Young University, 435 CTB, Provo, UT 84602, USA
}

Correspondence should be addressed to J. Crockett, crockettj@byu.edu

Received 9 April 2012; Accepted 17 October 2012

Academic Editor: Sergej Zilitinkevich

Copyright ( 2012 B. Casaday and J. Crockett. This is an open access article distributed under the Creative Commons Attribution License, which permits unrestricted use, distribution, and reproduction in any medium, provided the original work is properly cited.

\begin{abstract}
Using ray theory, we explore the effect an envelope function has on high-frequency, small-scale internal wave propagation through a low-frequency, large-scale inertia wave. Two principal interactions, internal waves propagating through an infinite inertia wavetrain and through an enveloped inertia wave, are investigated. For the first interaction, the total frequency of the highfrequency wave is conserved but is not for the latter. This deviance is measured and results of waves propagating in the same direction show the interaction with an inertia wave envelope results in a higher probability of reaching that Jones' critical level and a reduced probability of turning points, which is a better approximation of outcomes experienced by expected real atmospheric interactions. In addition, an increase in wave action density and wave steepness is observed, relative to an interaction with an infinite wavetrain, possibly leading to enhanced wave breaking.
\end{abstract}

\section{Introduction}

Internal gravity waves exist abundantly in uniformly, stably stratified fluids, such as the ocean and atmosphere. Naturally occurring perturbations such as flow over topography [1-5], convective storms [6-11], and geostrophic adjustment [12$15]$ in the atmosphere continuously create internal waves. Observed internal waves have vertical wavelength scales from meters to kilometers and horizontal wavelengths of tens of meters to thousands of kilometers. These waves significantly affect flow dynamics in the atmosphere. Mixing induced by dissipating gravity waves in the atmosphere is important to the vertical transport of chemicals, energy, and momentum [16]. This momentum and energy transport plays a central role in driving the mean meridional circulation [17]. Other global circulation patterns in the middle atmosphere are driven by the drag and diffusion caused by internal wave breaking [18-21]. These include the quasibiennial oscillation of the equatorial lower stratosphere [22] and the semiannual oscillations of the equatorial upper stratosphere and mesosphere [23].
Several various mechanisms may lead to internal waves shifting or refracting to higher frequency and steepening, eventually leading to wave breaking. These include high-frequency wave-wave interactions [24, 25], high-lowfrequency wave interactions [26-31], wave-vortex interactions [32], self-acceleration [33,34], and wave steepening due to propagation through a shear [35-37].

Multiple authors [37-42] have explored internal wave propagation through a mean background wind in the form of a steady shear leading to turning points or critical levels. Turning points occur when the internal wave is propagating opposite to the background wind and it is refracted to the natural frequency of the fluid and then the wave must turn back on itself. Critical levels occur when the internal wave is propagating in the same direction as the background wind and the wave is refracted until its horizontal phase speed reaches the background wind speed. As the internal waves approach smaller vertical wavelengths in the critical level scenario, the low-amplitude waves are absorbed and the larger-amplitude waves break yet some of their energy may be transmitted above the critical level. Hines [43-46], 
Eckermann [28], Vanderhoff et al. [29, 30], Broutman and Young [26], Bruhwiler and Kaper [27], and Sartelet [47, $48]$ included time-dependence and spatial variability of the background wind, although still on long time scales and large length scales, in the form of an inertia wave. The result was a reduction in the probability of critical levels, which became time-dependent, and an introduction of another type of strong refraction, caustics.

Caustics occur when small-scale, high-frequency (short) waves are propagating opposite to a large-scale, lowfrequency (inertia) wave (common in the ocean) and the vertical group velocity of the short wave is equal to the vertical phase speed of the inertia wave. When this strong refraction occurs, the small waves experience quick changes in wavenumber and group velocity resulting in spreading the short wave wavenumbers and transferring energy with the inertia wave $[27,29,30,47-49]$. Although this type of scenario is possible in the atmosphere, it is less common and will not be discussed in depth here.

Small-scale waves propagating in the same direction as an inertia wave have been observed in the atmosphere [13, $50,51]$. Upward propagating envelopes of inertia waves with vertical wavelengths between 1 and $7 \mathrm{~km}$, horizontal wavelengths on the scale of $1000 \mathrm{~km}$, and maximum horizontal velocities of $3 \mathrm{~m} / \mathrm{s}$ were observed. Thompson [51] showed that the source of inertia waves is generally near the earth's surface, propagating upward and thus inertia waves are commonly found in the stratosphere. Since internal waves are constantly being generated in the troposphere near the earth's surface, the interaction between upward propagating internal waves and inertia waves is expected to be a regular occurrence. Sato et al. [50] found that other background winds in the vicinity of the inertia waves were relatively small such that the shear due to the inertia wave dominates short wave refraction.

Eckermann [28] found that small waves interacting with an infinite inertia wavetrain propagating the same direction did not encounter a critical level since the oscillations from the background flow did not cause the relative frequency of the small wave to decrease toward zero at any particular location, but rather caused the small wave frequency to oscillate as the wave propagated through phases of the inertia wave. The small waves did, however, reach critical wavenumbers. When the short wave vertical wavenumber becomes large relative to the initial wavenumber or fluid environment, waves are assumed to overturn and be dissipated by turbulence. This is referred to as the Jones critical level and was defined in Eckermann's work as 12.5 meters. The waves in a time-dependent shear were also less likely to reach a turning point, compared with steady shear. Sartelet $[47,48]$ showed that, with an infinite series of enveloped inertia waves, correlating to Eckermann's research, these waves would all follow similar trajectories as Eckermann found.

This paper will investigate the interaction between small-scale, high-frequency internal wave packets and timedependent shear in the form of an inertia wave, which will be confined to a single envelope. We predict the possibility of critical levels and turning points based on the relative initial short wave properties and inertia wave, with specific interest in the effect the inertia wave envelope has on the dynamics of the interaction. Comparisons to the interaction between a short wave and infinite inertia wavetrain are made. In addition, differences in wave amplitude and steepness are discussed. Interactions will principally adopt atmospheric conditions, where the buoyancy frequency is constant throughout the interaction and both waves are propagating in the same direction.

Section 2 describes the problem setup. Section 3 describes the methods used for analysis. Section 4 includes results and discussion of wave propagation through a series of background shear profiles. In Section 5, we make conclusions and discuss the impact of these results.

\section{Idealized Problem}

2.1. Inertia Wave. The large-scale inertia gravity wave is idealized as a sinusoidal wave that exists either as an infinite wavetrain or as a finite wave contained within a Gaussian envelope. The frequency of the wave is equal to the Coriolis frequency $(f)$, resulting in an infinite horizontal wavelength and no vertical group speed. This assumption is supported by observed scales of inertia wavelengths being three orders of magnitude larger in the horizontal than vertical. In this work, $f=0.0001 \mathrm{~s}^{-1}$ and is assumed constant. The phases of the inertia waves propagate downward at a speed $C=f / M$, where $M$ is the vertical wavenumber of the wave and we keep with standard internal-wave notation [52] such that when $M<0$ the phases are propagating downward. The horizontal current resulting from the inertia wave, $(U, V, 0)$, is

$$
U+i V=G(z) U_{0} e^{i(M z-f t)},
$$

where subscript " 0 " defines an initial value and the amplitude $U_{0}$ is constant. The coordinate system is $(x, y, z)$ with $z$ positive upwards. $G$ is unity for an infinite wavetrain, and for a finite wave it is the envelope function:

$$
G=e^{-z^{2} / L^{2}},
$$

where the envelope scale $L$ is constant. Figure 1 shows the difference between the vertical structure of an infinite wavetrain and an enveloped inertia wave.

2.2. High-Frequency Internal Waves. The short waves are modeled as energy concentrated at a single point moving through space and time, along rays (discussed in next section). Their wavenumber $(k, 0, m)$ and intrinsic frequency $\hat{\omega}$ are related by the internal wave dispersion relation:

$$
\widehat{\omega}=\frac{\left(k^{2} N^{2}+m^{2} f^{2}\right)^{1 / 2}}{\left(k^{2}+m^{2}\right)^{1 / 2}} .
$$

Here, $N=\left[\left(-g / \rho_{0}\right)\left(d \rho_{0} / d z\right)\right]^{1 / 2}$ is the mean buoyancy frequency, assumed constant at $N=0.02 \mathrm{~s}^{-1}$, with $\rho_{0}$ as the mean density and $g$ as gravity. The vertical group speed of the short wave is

$$
c_{g z} \equiv \frac{\partial \widehat{\omega}}{\partial m}
$$




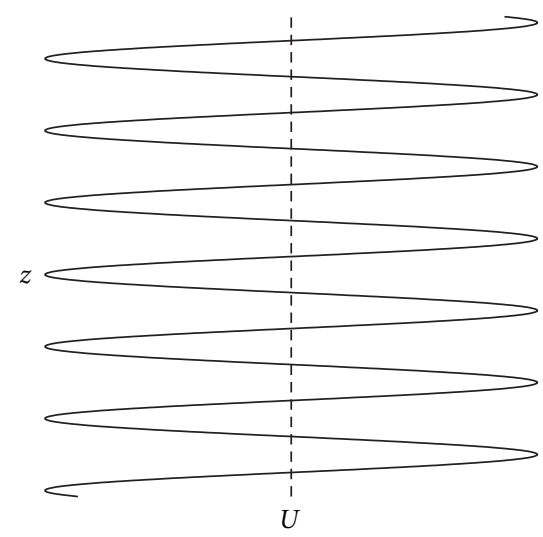

(a) Infinite wavetrain

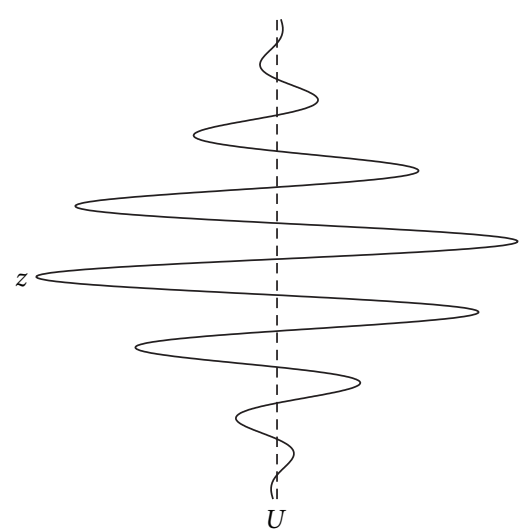

(b) Enveloped wave

FIGURE 1: Inertia wave velocity profiles used in this study. The plotted variable is $U(z, t=0)$ of (1), plotted here in nondimensional units. (a) $G=1$. (b) $G=e^{-z^{2} / L^{2}}$.

In this paper, $c_{g z}$ is always positive. The short waves approach the inertia wave envelope from below.

The frequency relative to the reference frame $(x, y, z)$ is

$$
\omega=\widehat{\omega}+k U
$$

and frequency relative to the inertia wave reference frame $(x, y, z-C t)$ moving vertically at speed $C$ is

$$
\Omega=\widehat{\omega}+k U-C m .
$$

In the inertia wave reference frame, the inertia wave phase $\xi=M z-f t$ is stationary and the change in $\Omega$ through time is defined as

$$
\frac{d \Omega}{d t}=G_{z} U_{0} C k \cos \xi
$$

For an infinitely long inertia wavetrain $(G=1), U$ and $V$ are steady in the inertia wave reference frame, and $\Omega$ is, therefore, constant following a short-wave ray [26]. However, when the inertia wave is confined to an envelope as in (2), $\Omega$ is no longer constant.

Here, we define a shift in apparent background velocity as the difference between the actual local background velocity and the predicted velocity assuming an infinite wavetrain (such that $\Omega=$ constant),

$$
U_{\text {zero }}=\frac{\Delta \Omega}{k},
$$

where $\Delta \Omega=(d \Omega / d t) \Delta t$ for small time steps. For simulations involving an infinite wavetrain $\left(G_{z}=0\right)$ or a timeindependent background flow field $(C=0)$, this value remains constant at $U_{\text {zero }}=0$.

Wave breaking can be estimated to occur when isopycnals are vertical, $\zeta_{z} \geq 1[47,48]$, where $\zeta=\zeta_{0} \exp (i(k x+m z-$ $\hat{\omega} t))$ is the vertical displacement of the short waves and $\zeta_{z}$ is the wave steepness. The subscript $z$ represents the partial derivative with respect to $z$. Wave steepness can be calculated using the dispersion relation and knowing the wave energy density as a function of wave steepness [30]:

$$
E=\frac{1}{2} \rho_{0} \zeta_{0}^{2} N^{2}\left[1+\left(\frac{f m}{N k}\right)^{2}\right] .
$$

Using the dispersion relations and (9) and recognizing from the equation for steepness that $\left|\zeta_{z}\right| \approx|m \zeta|$, the wave steepness can be calculated as

$$
\zeta_{z}=-m\left|\left(\frac{2 A \widehat{\omega}}{\rho_{0}}\right)^{1 / 2} N^{-1}\right|,
$$

where $A=E / \widehat{\omega}$ is the wave action density. Since the total wave action of an internal wave is constant, the wave action density will vary inversely as the volume of a set of nearby rays (ray tube) varies. However, since there are no changes in the $y$-dimension, no change in $k$, and the inertia wave is two-dimensional, the only dimension in which the ray tube volume will change is $z$. Defining $z_{0}$ as the initial dimension of the ray tube in $z, z / z_{0}=c_{g z} / c_{g z 0}$ and

$$
\frac{A}{A_{0}}=\frac{c_{g z 0}}{c_{g z}} .
$$

\section{Methods}

3.1. Ray Theory. To use ray theory, internal wave propagation must be of small amplitude (linear), and the Wentzel, Kramer, Brillouin, Jeffreys (WKBJ) approximation (slowly varying short wave parameters, and the large wave is unaffected by the interaction) must hold, and the small waves are defined by a single frequency (infinite wavetrain). The ray-tracing results in this paper are obtained with the following pair of ray equations, for the vertical position of the ray path and the vertical wavenumber, respectively,

$$
\frac{d x}{d t}=c_{g}+U, \quad \frac{d m}{d t}=-k \frac{\partial U}{\partial z} .
$$


Here, $d / d t=\partial / \partial t+c_{g} \cdot \nabla$. Because the expression (1) has no dependence on $x$ or $y$, the horizontal components $(k, 0)$ of the wavenumber of the short waves are conserved along the ray. These equations are solved using a fourth-order RungeKutta method.

Wave action density calculations are made using action conservation techniques and incorporating ray tube volume analysis. As the volume of the ray tube decreases, the wave action density (and amplitude) increases proportionally. Thus, an estimate of ray tube volumes furnishes the wave amplitude. For further discussion of the theory, see Hayes [53] and for implementation see Broutman [49].

3.2. Estimating Turning Points and Critical Levels. Turning points occur where $\hat{\omega}=N$. The velocity at this location can be found by assuming $\omega$ and $\Omega$ are constant and substituting $\widehat{\omega}=N$ into (5) or (6) for steady and inertia wave backgrounds, respectively,

$$
\begin{gathered}
U_{t s}=\frac{\omega_{0}-N}{k}, \\
U_{t}=\frac{\omega_{0}-N-C m_{0}}{k} .
\end{gathered}
$$

Critical levels occur where $\widehat{\omega}=f$. Again, the velocity at this location can be found by substituting this condition into (5) or (6) for steady and inertia wave backgrounds, respectively. For the steady background, the velocity is

$$
U_{c s}=\frac{\omega_{0}-f}{k} .
$$

For the inertia wave background, the horizontal velocity of the inertia wave must approach infinity for $\hat{\omega}$ to approach $f$. Thus, a different type of critical level is defined. It is assumed that waves which reach a small wavelength are probably approaching breaking. We will use the defined wavelength, a typical critical value for the atmosphere, used by Eckermann [28] (following [54]) of 12.5 meters. Thus, $m_{c}=-2 \pi / 12.5 \mathrm{~m}^{-1}=200 \mu$, where $\mu$ is a scaling used for convenience. The inertia wave vertical wavenumber is held constant in throughout most of the study at $M=0.001 \mathrm{~m}^{-1}$. The inertia wave velocity at this critical level can be estimated as

$$
U_{c}=\frac{1}{k}\left(\omega_{0}^{1 / 2}-\omega_{c}^{1 / 2}\right)+\frac{f\left(m_{c}-m_{0}\right)}{M k},
$$

where $\omega_{c}$ is the frequency evaluated at the critical wavenumber, $m_{c}$.

\section{Results}

4.1. Interaction with an Infinite Inertia Wavetrain. Eckermann [28] tested their theory of the accuracy of Doppler spreading assumptions with small waves interacting with a steady background shear, a steady background with a wave form, and a propagating infinite inertia wavetrain. To remove the effect of the inertia wave velocity on the small wave initially, all small waves are initiated in regions where $U=0$.
Short waves are initiated well below the inertia wave envelope where $U=0$ and envelopes of stationary shear and inertia waves are both considered to distinguish between the effects of enveloping and time dependence. In addition, an analysis of wave stability is discussed.

For time-independent background shear, Eckermann [28] showed that in addition to critical levels for certain interactions, there exists a critical vertical wavenumber for the small wave. This means that for a range of short waves, with differing frequencies or wavenumbers, all waves with a vertical wavelength smaller than a critical value would encounter either a critical level or a turning point when approaching the time-independent background wave of specified amplitude. He confirmed that a critical level occurs when the frequency of the small wave approaches the local Coriolis frequency, and a turning point occurs when the frequency approaches the local buoyancy frequency.

Using (13) and (15), the outcomes may be predicted for an interaction between a small internal wave and a large inertia wavetrain. Figure 2 shows the potential outcomes of a wide range of waves interacting with inertia wavetrains of various amplitudes. The format is similar to the plots of Eckermann [28] and shows interactions with infinite wavetrains of different maximum background velocities: (a) $1.5 \mathrm{~m} / \mathrm{s}$, (b) $5.0 \mathrm{~m} / \mathrm{s}$, and (c) $8.3 \mathrm{~m} / \mathrm{s}$, respectively. The horizontal and vertical axes define the nondimensional initial short wave vertical wavenumber and frequency, respectively. In all cases, $f, N$, and the vertical wavenumber of the inertia wave, $M=-0.001 \mathrm{~m}^{-1}$, were kept constant. Since $k$ also will remain constant throughout the interaction, lines of constant $k$ are displayed in Figure 2(a). The green triangles represent interactions where the small wave will reach a turning point, the red squares represent a critical level interaction (based on a critical wavenumber), and the blue circles represent either a critical level or a turning point depending on whether the wave propagates first through positive or negative background velocities. The other dots without surrounding shapes represent regions of free propagation of the small waves. It is clear that an increase in the inertia wave amplitude increases the critical level and turning point regions.

In Figure 2, the left vertical black line indicates where the initial vertical wavelength of the short wave matches the vertical wavelength of the inertia wave. Any simulations near or to the left of this line are not scale-separated and the results may not be completely accurate. The vertical black line on the right indicates the value of the critical wavenumber, and any data to the right of this line is initialized in the Jones critical level. These waves are, therefore, assumed to become unstable due to any interaction with the background. Therefore, only data between these lines should be considered valid for these simulations.

To estimate changes in wave action density, (11) is used in the frame of reference moving with the inertia wave phases such that $d z / d t=c_{g z}-C$, and it is found, in agreement with [28],

$$
\frac{A}{A_{0}}=\frac{c_{g z 0}-C}{c_{g z}-C}
$$




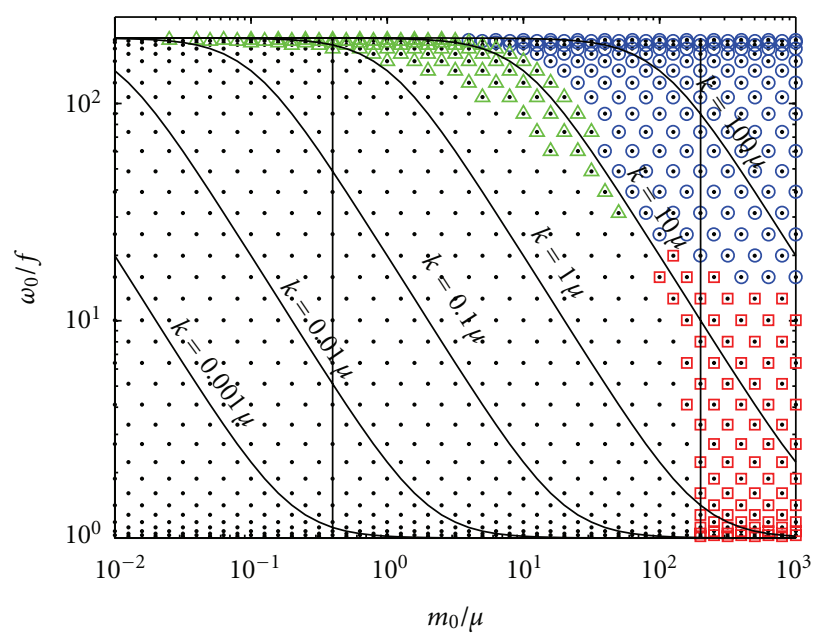

(a) $U_{0}=1.5 \mathrm{~m} / \mathrm{s}$

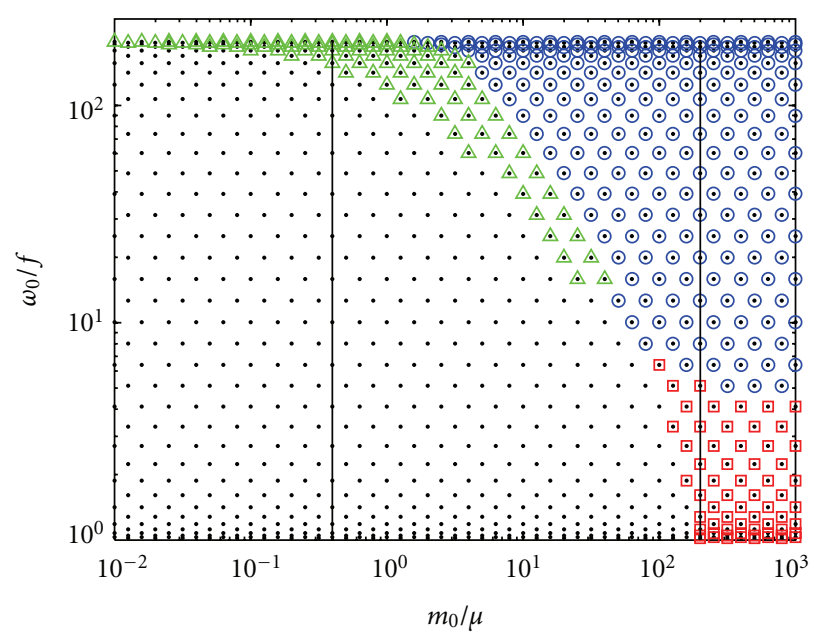

(b) $U_{0}=5.0 \mathrm{~m} / \mathrm{s}$

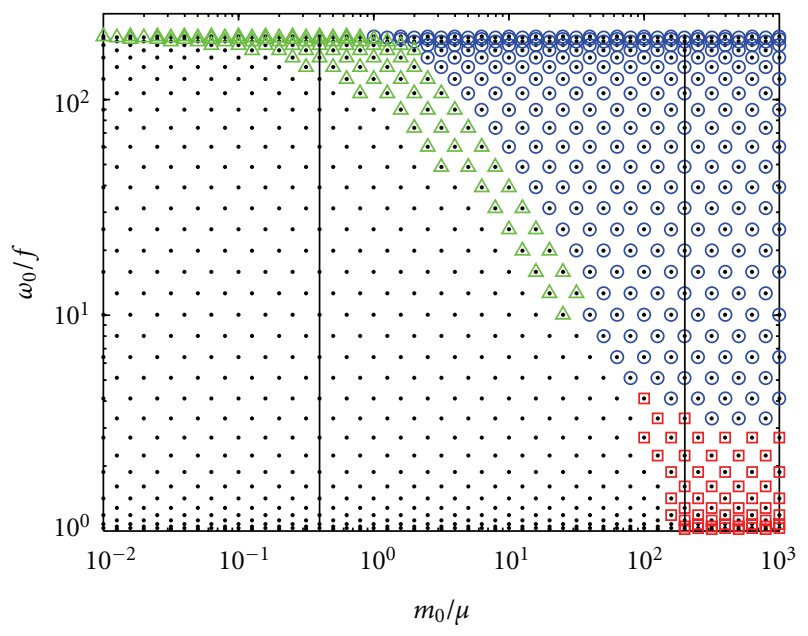

(c) $U_{0}=8.3 \mathrm{~m} / \mathrm{s}$

Figure 2: Probability of short waves reaching a turning point (green triangles), critical level (red squares), or either (blue circles) during propagation through an infinite inertia wavetrain. Lines on (a) represent constant $k$ as labeled. Superimposed vertical lines depict wavenumber limits as discussed in the text.

Equation (17) indicates that there is a maximum change associated with an infinite velocity and therefore an infinite vertical wavenumber. This is done by setting $U=\infty$ and subsequently, $c_{g z}=0$. Percentage changes in wave action density for two scenarios with different inertia wave amplitudes and phase speeds are displayed in Figure 3, where the colors represent the scale of the change as a function of the initial frequency and wavenumber of the short wave. The waves that will experience the largest change in wave action density are those that have initial frequencies near $N$ and initial vertical wavenumbers between $\mu$ and $10 \mu$. We have found the region is bounded by limits inherent to the interaction, a steady critical level estimate and the inertia wave phase speed, which can be seen as the white lines in Figure 3. The lower limit bounds the very low wavenumber waves, which would not reach a critical level in a time-independent flow field with the same velocity and thus do not have a significant change in wave action density even when the background is timedependent. The upper limit bounds the short waves initially propagating upward faster than the phases of the inertia wave propagate.

The wave action density influences the probability of wave breaking as the wave steepness is also a function of the short wave amplitude. An increase in wave action density results in an increase in wave steepness. Rearranging (10) and normalizing by the initial value,

$$
\frac{\zeta_{z}}{\zeta_{z 0}}=\left(\frac{A \omega_{0}}{A_{0} \omega}\right)^{1 / 2} .
$$

Wave steepness depends on the frequency and action density, with the maximum steepness occurring when the action density is a maximum and the frequency is a minimum. The maximum changes in wave frequency occur for waves with the largest initial vertical wavenumbers and frequencies. These waves are far enough from the critical level velocity and Coriolis frequency to support significant changes in frequency. For these interactions, the largest change in wave 


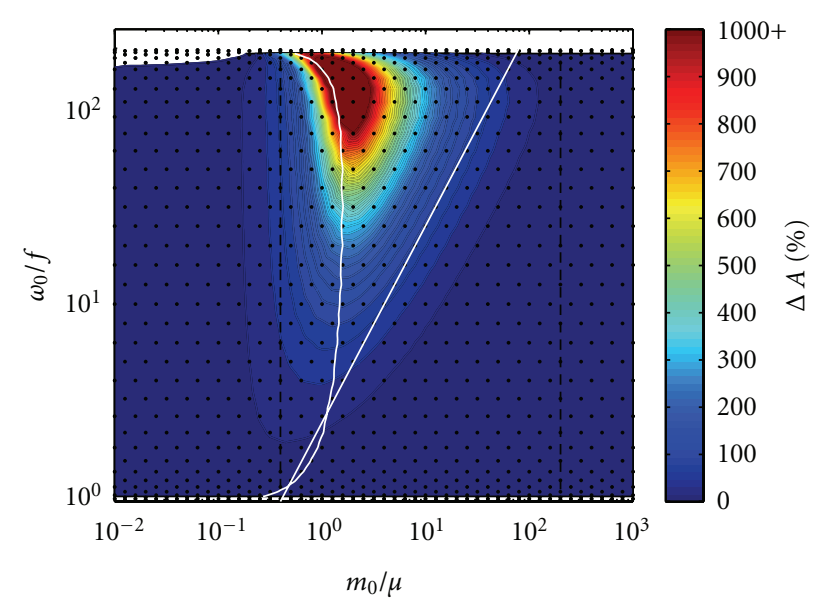

(a) $U_{0}=5.0 \mathrm{~m} / \mathrm{s}, C=0.1 \mathrm{~m} / \mathrm{s}$

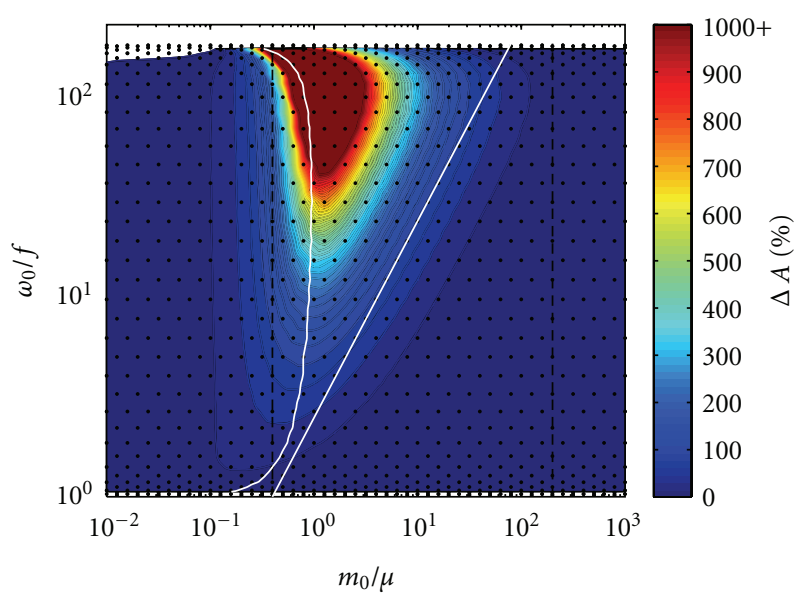

(b) $U_{0}=1.5 \mathrm{~m} / \mathrm{s}, C=0.01 \mathrm{~m} / \mathrm{s}$

FIGURE 3: Contours of maximum change in wave action density for two scenarios with different background velocities and inertia phase speeds. The white line to the left shows the critical level boundary for a stationary background, and the white line to the right shows the value of the inertia wave phase speed.

action density and wave steepness both occurs where the background velocity is at a maximum positive value.

The contours in Figure 4 show the maximum change in wave steepness calculated using (18). The most severe change in wave steepness in Figure 4 occurs for waves that have large initial frequencies and initial vertical wavenumbers between $\mu$ and $10 \mu$, which corresponds to the wave action density shape changes as well. This region increases for increasing background velocities, as can be seen when comparing Figures $4(\mathrm{a})$ and $4(\mathrm{~b})$. When internal waves reach excessive wave steepness, defined by a value of unity according to (10), the waves become unstable and are likely to overturn, break, and be dissipated by turbulence. Because wave steepness is given as a relative change, it would be necessary to know an initial steepness of the internal waves to predict whether they would overturn due to excessive wave steepening.

It should be noted that for linear ray theory, the amplitude, which affects the initial wave steepness, has no effect on the probability of reaching a critical wavenumber or reflection, so each potential outcome is independent of the other. Figure 4 illustrates the change in wave steepness jointly with the waves that will experience a critical level or turning point for two different inertia wave amplitudes, showing how the same waves may steepen without approaching a critical level. In the regions where waves will experience large changes in wave steepness, those waves may be otherwise expected to freely propagate or become reflected.

As the phase speed of the inertia wave decreases, it approaches a time-independent flow field. Thus, the probability of the Jones critical level increases. Incidentally, the probability of turning points also increases. The alternate is also true, in that if the inertia wave phase speed increases, the probability of a critical level or turning point decreases. As the phase speed of the inertia wave increases, the small wave has less time to be affected by the interaction, ultimately reducing the severity of the interaction.
4.2. Interaction with an Enveloped Inertia Wave. In the previous scenarios, it was theoretically possible to determine the wavenumbers, frequency, and wave action density of the short waves for any given background velocity and initial wave properties through the constancy of the total frequency. This is not the case for an interaction with an enveloped inertia wave (here, there are approximately six wavelengths contained within the envelope). During the interaction, the positively propagating short waves will spend less time in negative background velocities and more time in positive background velocity, due to the vertical group velocity of the small waves growing in positive shear and decreasing in negative shear.

During the first half of the interaction (bottom half of envelope where $G_{z}>0$ ), the background velocity magnitudes are diminishing as they propagate downwards through the inertia wave envelope $(C<0)$, and as such the total frequency, $\Omega$, of the short wave will decrease (7). Similarly, as the wave propagates out of the envelope (top half of envelope where $G_{z}<0$ ), the total frequency increases towards its original value prior to the interaction. Assuming that a critical level or reflection do not occur, the short waves propagate out of the inertia wave envelope with no permanent changes.

Figures 5 and 6 show how $m$ and $\zeta$, respectively, change with the local background velocity as the short wave propagates. The short wave is initialized with $m_{0} / \mu=10$ and $k / \mu=$ -1 and the inertia wave has a maximum background velocity of $5 \mathrm{~m} / \mathrm{s}$. Figures $5(\mathrm{a})$ and $6(\mathrm{a})$ are interactions with an infinite wavetrain, and Figures 5(b) and 6(b) are interactions with an inertia wave contained within a Gaussian envelope. The small wave and inertia wave properties are identical to those defined for Figure 2(b), with the only difference being that Figure 5(b) involves an inertia wave envelope. The oscillations of $m$ with inertia wave velocity in Figure 5(a) cannot be seen, as they lay directly on top of each other as 


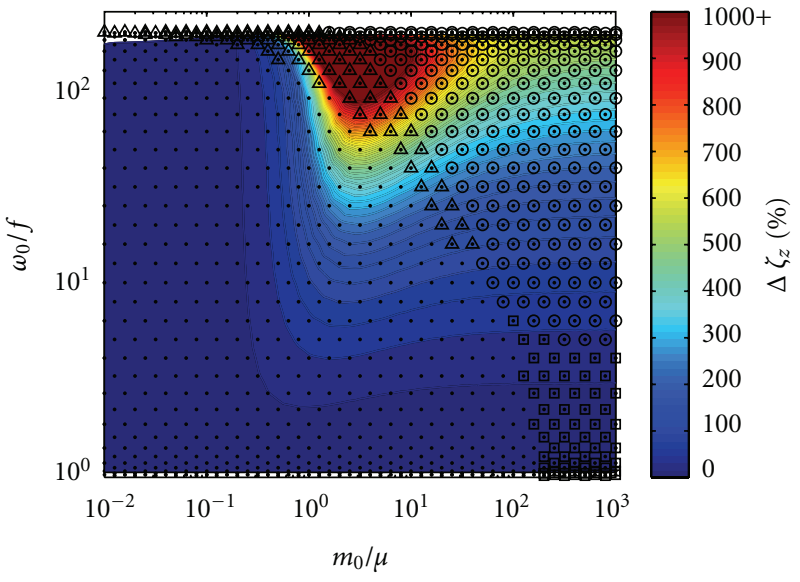

(a) $U_{0}=5.0 \mathrm{~m} / \mathrm{s}$

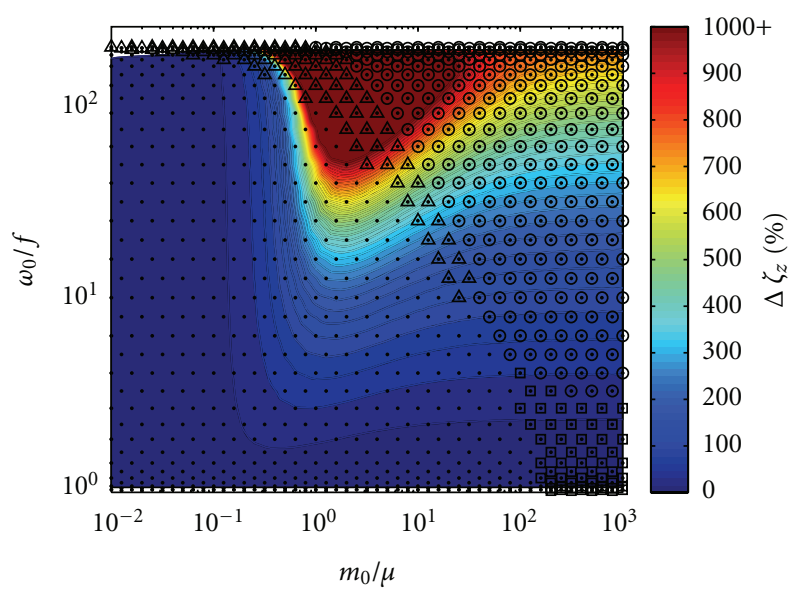

(b) $U_{0}=8.3 \mathrm{~m} / \mathrm{s}$

Figure 4: Contours of percentage changes in short wave steepness and symbols for expected turning points (green triangles), critical levels (red squares), and both (white circles).

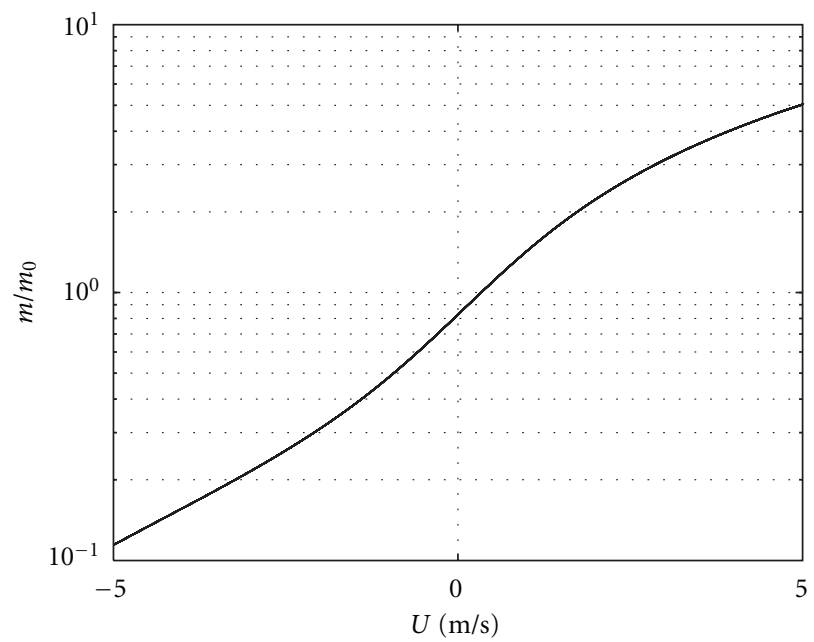

(a) Infinite inertia wavetrain

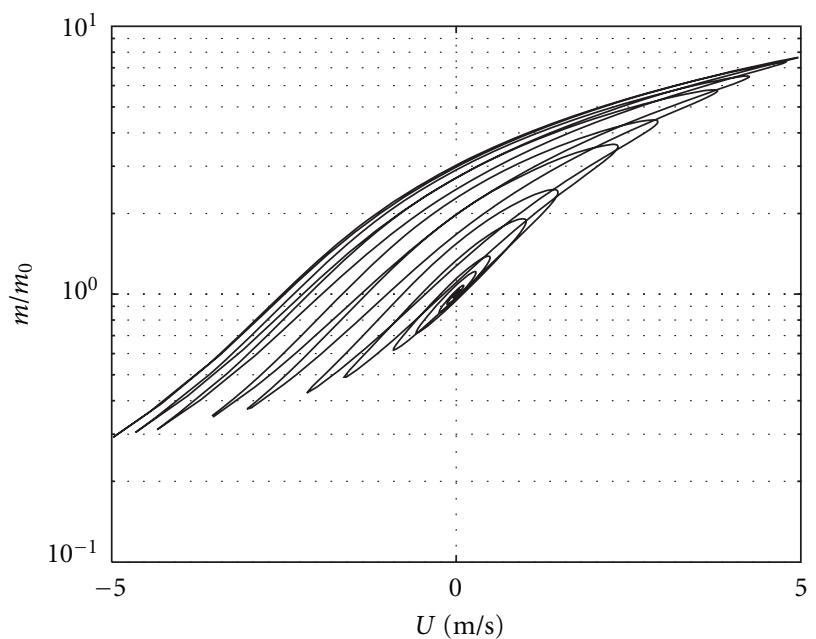

(b) Enveloped inertia wave

FIGURE 5: Normalized vertical wavenumber of a short wave with $m_{0} / \mu=10$ and $k / \mu=-1$ as it propagates through an inertia wave with $U_{0}=5 \mathrm{~m} / \mathrm{s}$.

the wave oscillates within the phases of the infinite wavetrain. However, a shift is seen in Figure 5(b) as the line is followed from $m / m_{0}=0, U=0$ along the lines until at largest spread of velocity $\mathrm{m} / \mathrm{m}_{0}$ is oscillating between approximately 0.3 and 8. At the beginning of the simulation, $m$ only matches its initial value with background velocities near zero. During the interaction with the inertia wave envelope, $m$ passes its initial values with background velocities below zero. The largest shift occurs at the center of the envelope, where $\mathrm{m} / \mathrm{m}_{0}$ ranges from approximately 0.3 to 8 instead of 0.15 to 5 . The curves in Figure 5(b) are nearly parallel to the curves in Figure 5(a), only shifted along the horizontal axis. One can see near the center of the envelope (region with largest oscillations), where $\mathrm{m} / \mathrm{m}_{0}=0, U=-2.5$ instead of zero. This is what we designate as $U_{\text {zero }}$. Thus, the effective background velocity ranges from $-2.5 \mathrm{~m} / \mathrm{s}$ to $7.5 \mathrm{~m} / \mathrm{s}$ when comparing to the theory of an infinite wavetrain. It will be shown that this results in a higher probability for critical levels and a lower probability for turning points. In addition, the waves have a larger steepness (Figure 6) for a longer period of time during the envelope. This may increase the possibility of wave breaking.

The effective total background velocity, $U+U_{\text {zero }}$, can be calculated using the $m$ values determined through ray tracing and (6) and (8) as follows:

$$
\widehat{\omega}_{0}-C m_{0}+U_{\text {zero }} k=\widehat{\omega}+U k-C m .
$$

Rearranging,

$$
U_{\text {zero }}=\frac{C\left(m_{0}-m\right)+\left(\hat{\omega}-\widehat{\omega}_{0}\right)}{k}+U .
$$




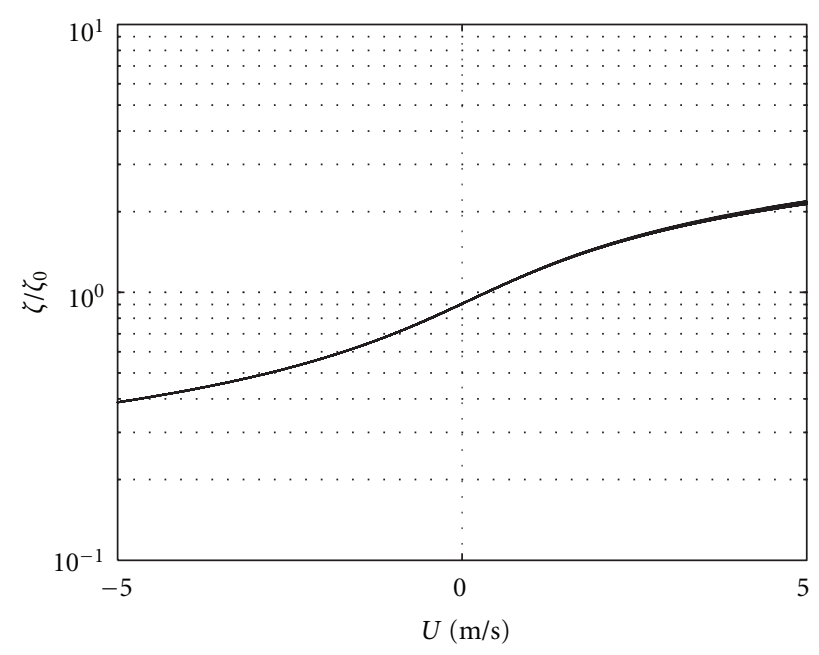

(a) Infinite inertia wavetrain

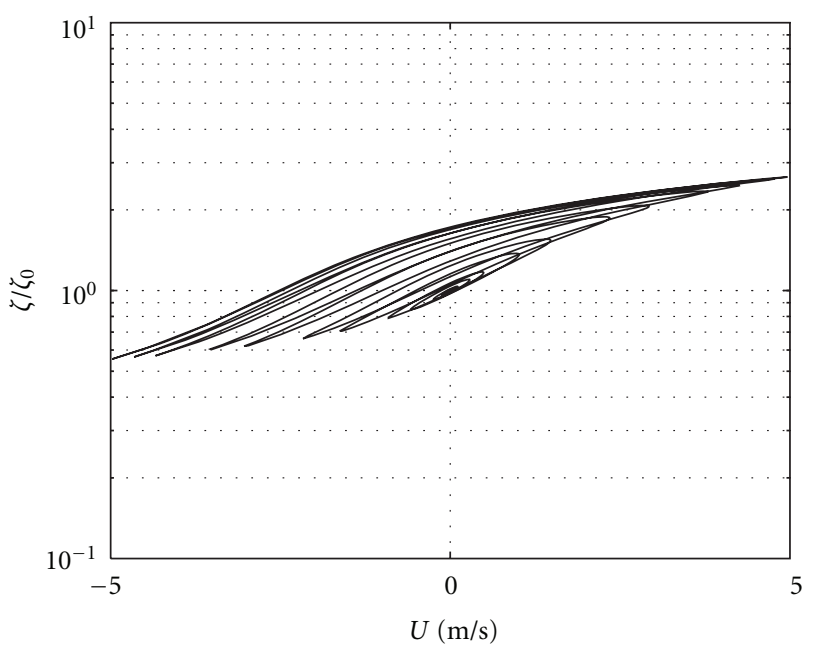

(b) Enveloped inertia wave

FIGURE 6: Normalized steepness of a short wave with $m_{0} / \mu=10$ and $k / \mu=-1$ as it propagates through an inertia wave with $U_{0}=5 \mathrm{~m} / \mathrm{s}$.

Using (7) and (8), changes in $U_{\text {zero }}$ over time can be calculated:

$$
\frac{d U_{\text {zero }}}{d t}=G_{z} U_{0} C \cos \xi .
$$

The value of $U_{\text {zero }}$ can also be represented relative to the size of the envelope, $U_{e}=G U_{0}$,

$$
\frac{d U_{\text {zero }}}{d U_{e}}=\frac{C}{c_{g z}} \cos \xi .
$$

Because $c_{g z}$ is a function of $m$ in these scenarios, and $m$ is a function of both $U$ and $U_{\text {zero }}$, the analytical solution to this equation is nonlinear and difficult to display, though the trend can be seen in Figures 7(b) and 7(d), where the black lines oscillate as the short waves propagate through the inertia wave.

Figures $7(a)$ and $7(\mathrm{c})$ show the evolution of the rays through the inertia wave, where the shaded regions are outlined by velocities at which the short wave would theoretically reach a critical level. Figures $7(\mathrm{~b})$ and $7(\mathrm{~d})$ display the corresponding shift in horizontal velocity, $U_{\text {zero }}$ for the interaction. The small wave and inertia wave properties are identical to those defined for Figure 2(b), with the only difference being that Figure 7 involves an inertia wave envelope. Figures 7 (c) and 7(d)show the same short wave propagating through an inertia wave with an envelope of twice the size. The value of $U_{\text {zero }}$ oscillates as the small waves propagate through the phases of the inertia wave, indicated by the black lines in $(b, d)$, but the average change is nearly hyperbolic and is not a function of the envelope length scale, $L$. For a larger inertia wave envelope, the magnitude of the oscillations of $U_{\text {zero }}$ diminish because the inertia envelope opens and closes more slowly. Because the magnitude of the oscillations is inversely related to $L$, or rather the magnitude is related to the shear due to the envelope, waves may be slightly more likely to experience a critical level or turning point sooner when interacting with inertia waves with smaller envelope. For a Gaussian envelope, $d U_{e} / d z$ is near zero at locations near the center of the envelope, and the $U_{\text {zero }}$ oscillations diminish in these regions, as indicated by Figures $7(\mathrm{~b})$ and $7(\mathrm{~d})$. This indicates that the total probability of small waves reaching a critical level or turning point does not significantly change with different envelope scales.

Notice the largest shift occurs near the center of the envelope. At this point, the wave no longer experiences background wind oscillations equal to the maximum velocities of an infinite inertia wave, but rather the background velocities offset by $U_{\text {zero }}$. In Figure 7, the short wave is propagating through an inertia wave with maximum oscillations of $5 \mathrm{~m} / \mathrm{s}$, and the short wave experiences a shift of $-2.5 \mathrm{~m} / \mathrm{s}$; so the short wave is experiencing oscillations of 7 to $-2.5 \mathrm{~m} / \mathrm{s}$, as discussed previously.

Figure 8 shows how $U_{\text {zero }}$ will affect turning points and critical levels during the interaction. For these simulations, $U_{0}=5 \mathrm{~m} / \mathrm{s}$. The probability of turning points has diminished, with no waves of initial frequency lower than approximately $30 f$ reaching one. Other waves, which would have required background velocities larger than $5 \mathrm{~m} / \mathrm{s}$ to reach a critical level interacting with an infinite wavetrain, reached critical levels. For all the waves tested in this interaction, the inertia envelope caused the number of waves that reached a critical level to increase by $4 \%$ and the number of reflections to decrease by $21 \%$. The number of freely propagating waves stayed constant at $60 \%$ of all waves tested.

In all these simulations, the inertia wave is contained within a Gaussian envelope, yet the shape of the envelope is not significant. Other scenarios were tested using triangular and parabolic envelopes and the average values of $U_{\text {zero }}$ were identical. Only the oscillating values of $U_{\text {zero }}$, illustrated in Figures 7(b) and 7(d), changed with the shape of the envelope. For the opening and closing of the envelope, (21) shows that a change in the envelope size corresponds to a specific change in $U_{\text {zero, }}$, and this governs the magnitude 


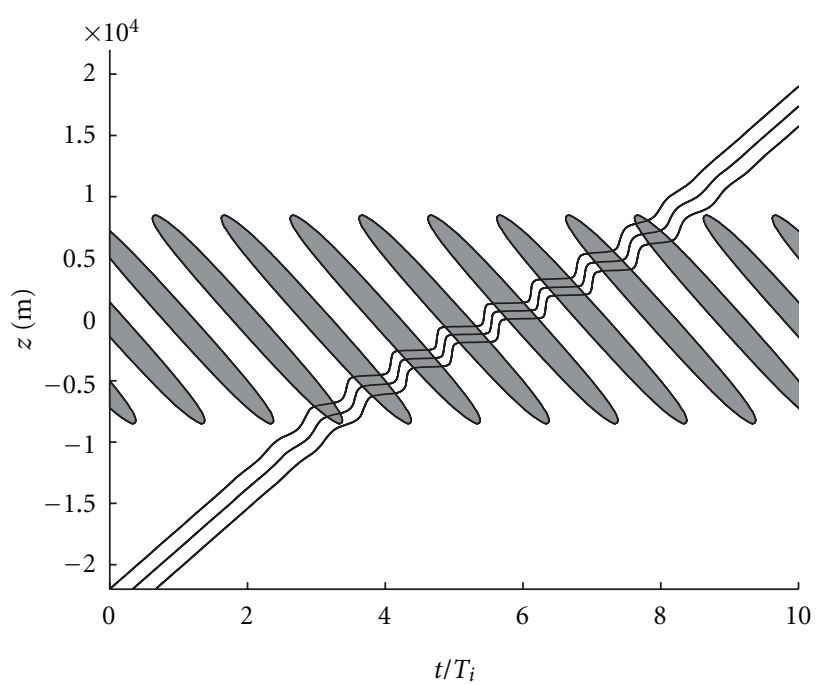

(a) Ray trajectories

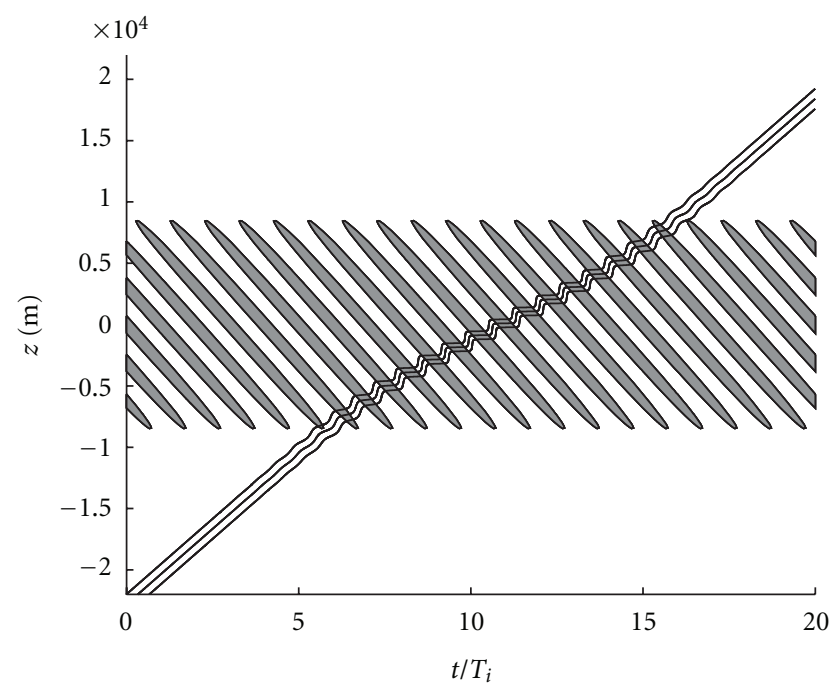

(c) As in (a) with $2 L$

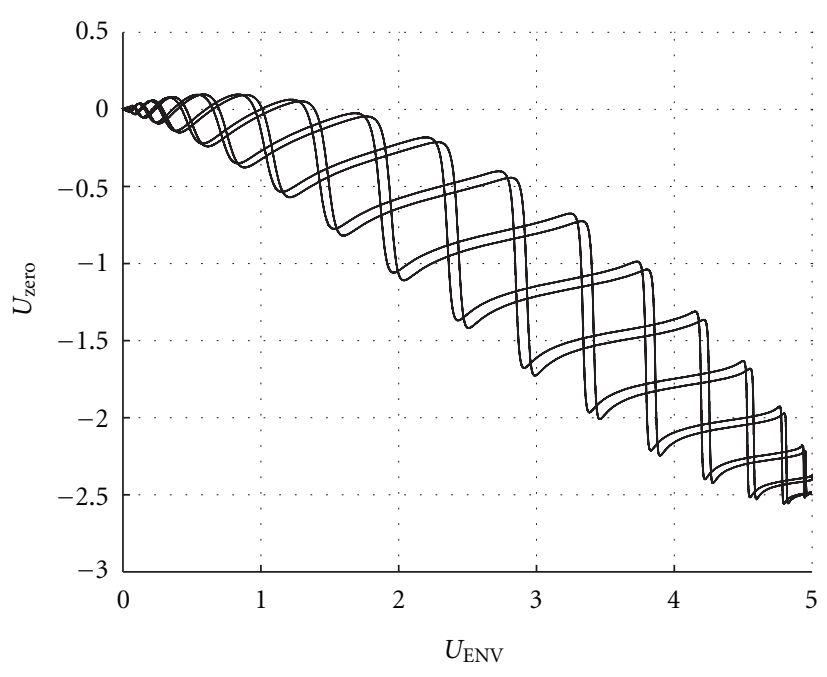

(b) Changes in $U_{\text {zero }}$

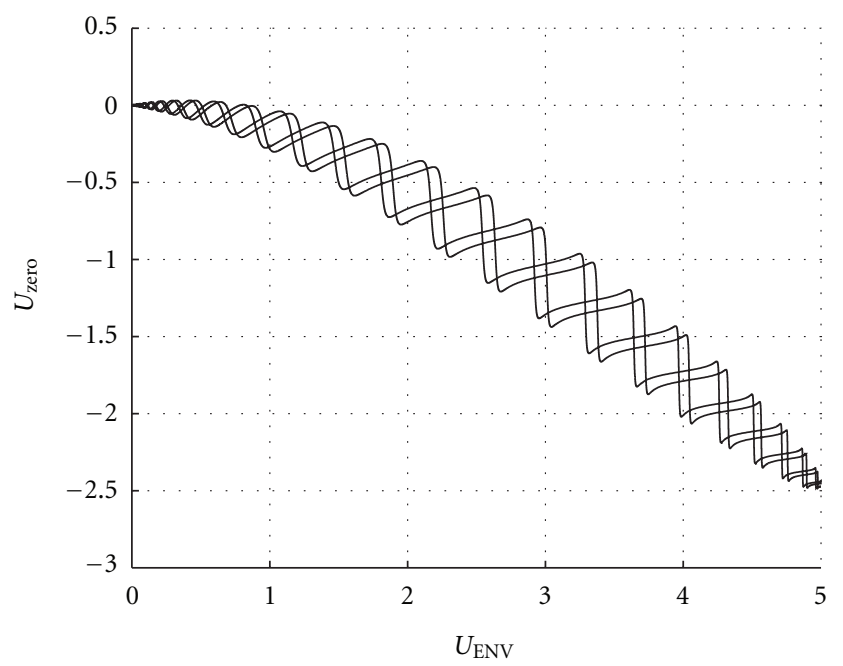

(d) As in (b) with $2 L$

FIGURE 7: Estimate of $U_{\text {zero }}$ along rays due to short wave propagation through an enveloped inertia wave. In (a), the shaded regions represent horizontal velocities exceeding time-independent critical level velocity. In (b), the black lines show $U_{\text {zero }}$ for each ray, one as each ray propagates into the center of the envelope, and the other as each propagates out of the envelope. The line through the center of the oscillations shows the average $U_{\text {zero }}$ during propagation. It begins at zero and decreases toward the center of the envelope, then returns to zero as is exits the envelope.

of the oscillations. It is expected that an integration over the envelope function $G_{z}$ would result in averaging out the oscillations and merely the trend to maximum $U_{\text {zero }}$ results.

Figure 9 illustrates the maximum $U_{\text {zero }}$ that occurs with numerous interactions with an inertia wave envelope with a maximum background velocity of $5 \mathrm{~m} / \mathrm{s}$, and the vertical wavenumber of the inertia wave envelope ranges from $M=$ -0.0008 to $-0.003^{-1}$ (corresponding to vertical wavelengths ranging from 2000 to $8000 \mathrm{~m}$ ). Notice that the horizontal axis is now normalized by the vertical wavenumber of the inertia wave, $M$, and the colorbar is normalized by the initial velocity, $U_{0}$. The small waves may experience magnitudes of $U_{\text {zero }}$ up to $80 \%$ of the total amplitude, but this only occurs for large initial vertical wavenumbers of both the inertia and short waves and in the midfrequency region of the short waves. Waves with small initial wavenumbers, where $m_{0}<M$, experienced little or no offset. These waves also had large initial group speeds and required large velocities for reaching a critical level, so it is not expected that they would experience large changes in $U_{\text {zero }}$. The region above the upper black line in the figure and to the right of the vertical line represents the areas without valid offset data. The upper portion represents the region where the short waves experience reflection before a maximum offset value can be obtained, and the far right portion represents a region where the small waves are initialized in the Jones critical level. The general shape of the offset when accounting for these critical lines is constant for varying $M$. 


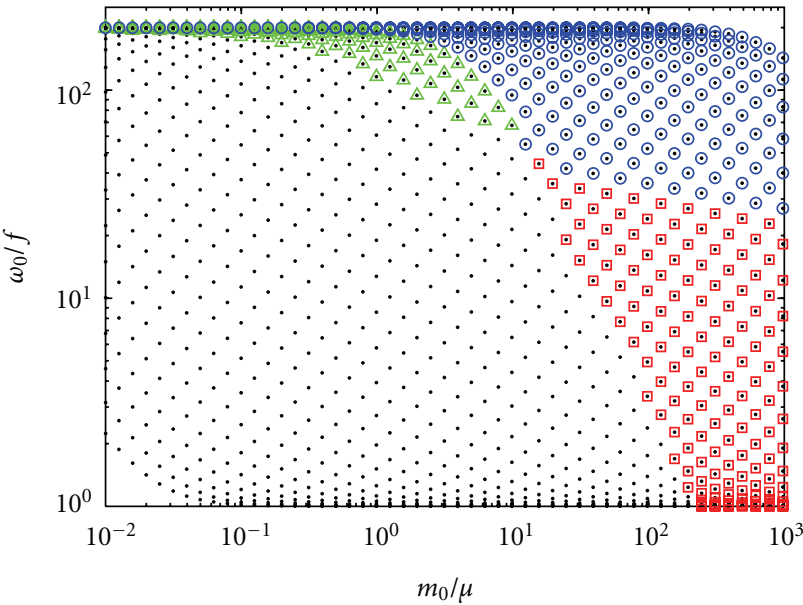

(a) Enveloped inertia wave

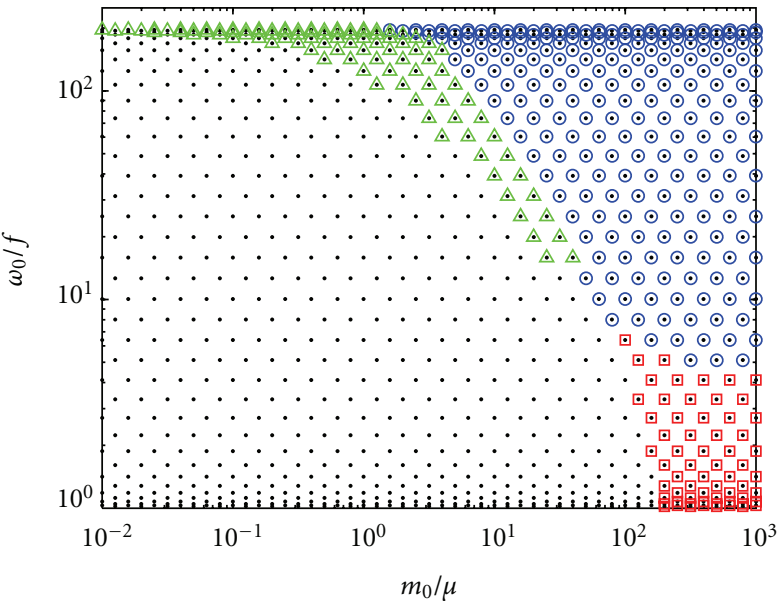

(b) Infinite inertia wavetrain

Figure 8: Possibility of turning points (green triangles), critical levels (red squares), or both (blue circles) due to short wave propagation through an enveloped inertia wave (a) and an infinite inertia wavetrain (b) with a maximum horizontal velocity of $5 \mathrm{~m} / \mathrm{s}$.

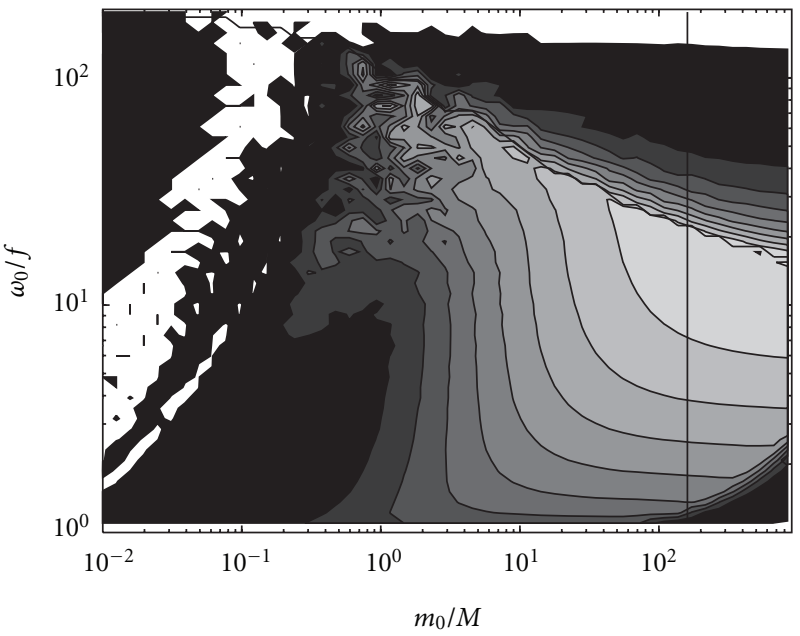

(a) $M=0.0031 / \mathrm{m}$

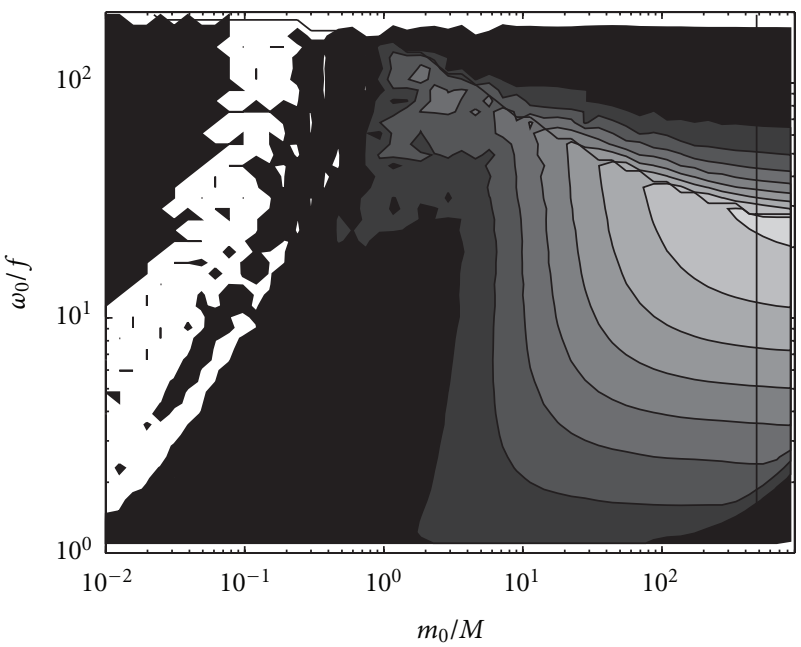

(c) $M=0.0011 / \mathrm{m}$

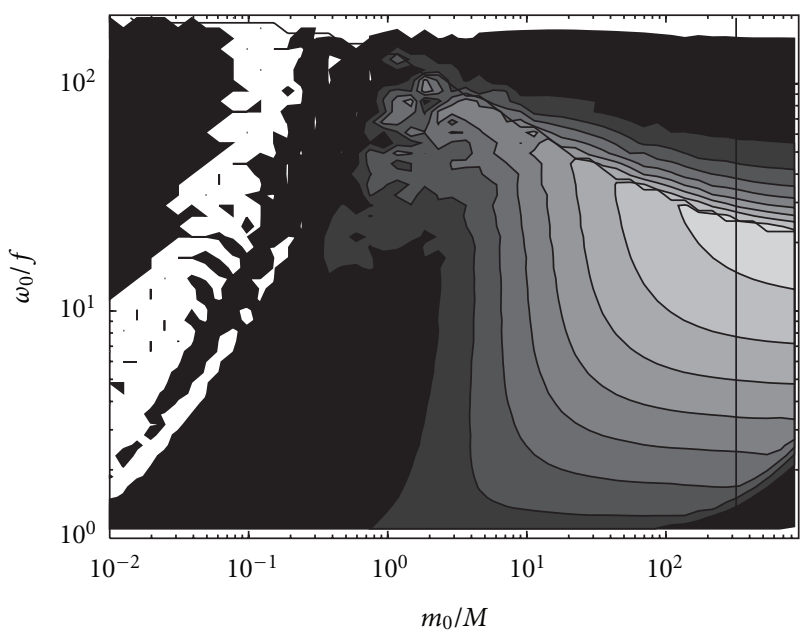

(b) $M=0.00161 / \mathrm{m}$

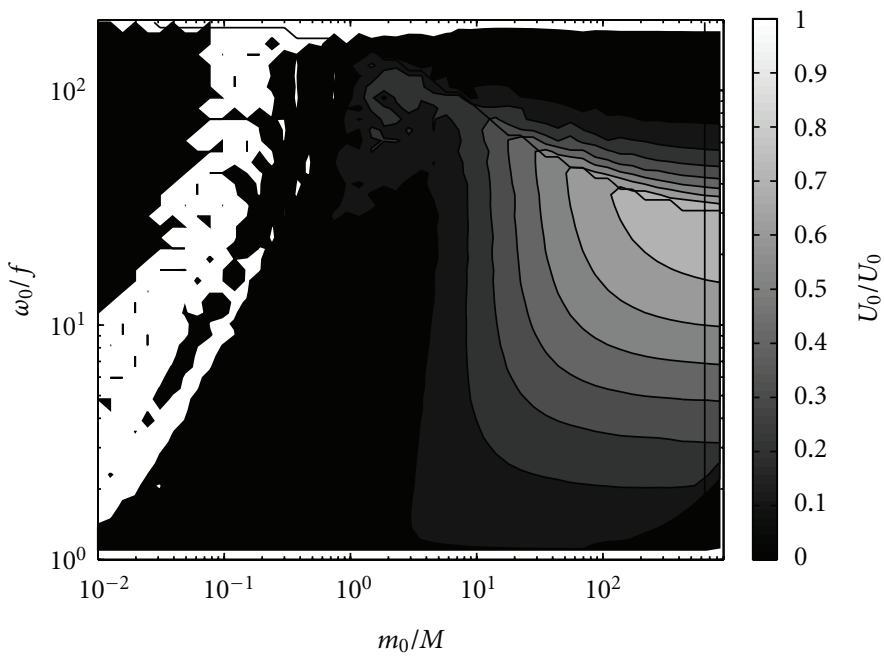

(d) $M=0.00081 / \mathrm{m}$

FIGURE 9: Maximum change in $U_{\text {zero }}$ for an interaction with an inertia wave envelope with $5 \mathrm{~m} / \mathrm{s}$ maximum horizontal velocity and varying $M$ values. 


\section{Conclusion}

The results of this paper examine the effects of enveloping an infinite inertia wavetrain, through which higher-frequency short waves propagate. Although the final effect of the envelope on short waves propagating though the inertia wave opposite to the phases should be minimal [29], during the interaction the envelope effectively forces more short waves toward critical levels, although these are now time-dependent and do not follow conventional means of approaching a steady critical level. In addition, turning points are significantly diminished due to the enveloping function. Thus in comparison with estimates of infinite inertia wave interactions [28], when the inertia waves are realistically enveloped, more high-frequency waves will deposit their energy at a lower altitude and more energy will continue to higher altitudes then will turn back downward.

The wave action density and wave steepness reach higher values for longer periods of time when the inertia wave is enveloped. These regions of increased amplitude were found to be bounded within the inertia wave by the steady critical level region and the inertia wave phase speed. These regions are then expected to be more likely to contain breaking waves.

When analyzing the effect of the envelope through a velocity shift experienced by the short waves, the relative shift is dependent on the relative vertical wavenumbers and background velocity (22). However, we have found that the shape and number of wavelengths within the envelope do not affect the velocity shift, merely the presence of the envelope causes the spatial dependence of the shear necessary for these alterations.

If current internal wave and global models account for this specific time-dependence and spatiality-dependence, they will more accurately predict critical levels, turning point locations, and wave amplitudes, improving estimations of when and where internal wave energy and momentum is transported and deposited in the atmosphere.

\section{Acknowledgment}

This material is based in part upon work supported by the National Science Foundation under Grant no. CBET0854131.

\section{References}

[1] K. R. Chan, L. Pfister, T. P. Buil et al., "A case study of the mountain lee wave event of January 6, 1992," Geophysical Research Letters, vol. 20, pp. 2551-2554, 1993.

[2] A. Dörnbrack, M. Leutbecher, R. Kivi, and E. Kyrö, "Mountain-wave-induced record low stratospheric temperatures above northern Scandinavia," Tellus, Series A, vol. 51, no. 5, pp. 951-963, 1999.

[3] M. Leutbecher and H. Volkert, "The propagation of mountain waves into the stratosphere: quantitative evaluation of threedimensional simulations," Journal of the Atmospheric Sciences, vol. 57, no. 18 , pp. 3090-3108, 2000.
[4] R. R. Long, "Some aspects of the flow of stratified uids, III, Continuous density gradients," Tellus, vol. 7, pp. 341-357, 1955.

[5] G. D. Nastrom and D. C. Fritts, "Sources of mesoscale variability of gravity waves. Part I: topographic excitation," Journal of the Atmospheric Sciences, vol. 49, no. 2, pp. 101-110, 1992.

[6] M. J. Alexander, J. H. Beres, and L. Pfister, "Tropical stratospheric gravity wave activity and relationships to clouds," Journal of Geophysical Research D, vol. 105, no. 17, pp. 2229922309, 2000.

[7] M. J. Alexander and L. Pfister, "Gravity wave momentum flux in the lower stratosphere over convection," Geophysical Research Letters, vol. 22, no. 15, pp. 2029-2032, 1995.

[8] E. M. Dewan, R. H. Picard, R. R. O’Neil et al., “MSX satellite observations of thunderstorm-generated gravity waves in mid-wave infrared images of the upper stratosphere," Geophysical Research Letters, vol. 25, no. 7, pp. 939-942, 1998.

[9] K. Sato, H. Hashiguchi, and S. Fukao, "Gravity waves and turbulence associated with cumulus convection observed with the UHF/VHF clear-air Doppler radars," Journal of Geophysical Research, vol. 100, no. 4, pp. 7111-7119, 1995.

[10] K. Sato, D. J. O'Sullivan, and T. J. Dunkerton, "Low-frequency inertia-gravity waves in the stratosphere revealed by threeweek continuous observation with the MU radar," Geophysical Research Letters, vol. 24, no. 14, pp. 1739-1742, 1997.

[11] R. A. Vincent and M. J. Alexander, "Gravity waves in the tropical lower stratosphere: an observational study of seasonal and interannual variability," Journal of Geophysical Research D, vol. 105, no. 14, pp. 17971-17982, 2000.

[12] D. C. Fritts and Z. Luo, "Gravity wave excitation by geostrophic adjustment of the jet stream. Part I: two-dimensional forcing," Journal of the Atmospheric Sciences, vol. 49, no. 8, pp. 681-697, 1992.

[13] F. M. Guest, M. J. Reeder, C. J. Marks, and D. J. Karoly, "Inertia-gravity waves observed in the lower stratosphere over Macquarie Island," Journal of the Atmospheric Sciences, vol. 57, no. 5, pp. 737-752, 2000.

[14] D. O’Sullivan and T. J. Dunkerton, "Generation of inertiagravity waves in a simulated life cycle of baroclinic instability," Journal of the Atmospheric Sciences, vol. 52, no. 21, pp. 36953716, 1995.

[15] S. L. Vadas and D. C. Fritts, "Gravity wave radiation and mean responses to local body forces in the atmosphere," Journal of the Atmospheric Sciences, vol. 58, no. 16, pp. 2249-2279, 2001.

[16] P. B. Hayes, J. F. Kafkalidis, W. R. Skinner, and R. G. Roble, "A global view of the molecular oxygen night glow," Journal of Geophysical Research, vol. 108, p. 4646, 2003.

[17] M. H. Hitchman, J. C. Gille, C. D. Rodgers, and G. Brasseur, "The separated polar winter stratopause: a gravity wave driven climatological feature," Journal of the Atmospheric Sciences, vol. 46, no. 3, pp. 410-422, 1989.

[18] M. J. Alexander and J. R. Holton, "A model study of zonal forcing in the equatorial stratosphere by convectively induced gravity waves," Journal of the Atmospheric Sciences, vol. 54, no. 3, pp. 408-419, 1997.

[19] J. T. Bacmeister, "Mountain-wave drag in the stratosphere and mesosphere inferred from observed winds and a simple mountain-wave parameterization scheme," Journal of the Atmospheric Sciences, vol. 50, no. 3, pp. 377-399, 1993.

[20] D. C. Fritts and M. J. Alexander, "Gravity wave dynamics and effects in the middle atmosphere," Reviews of Geophysics, vol. 41, no. 1, pp. 1-64, 2003. 
[21] C. J. Marks and S. D. Eckermann, "A three-dimensional nonhydrostatic ray-tracing model for gravity waves: formulation and preliminary results for the middle atmosphere," Journal of the Atmospheric Sciences, vol. 52, no. 11, pp. 1959-1984, 1995.

[22] M. Takahashi, N. Zhao, and T. Kumakura, "Equatorial waves in a general circulation model simulating a Quasi-BiennialOscillation," Journal of the Meteorological Society of Japan, vol. 75, no. 2, pp. 529-539, 1997.

[23] K. Hamilton and J. D. Mahlman, "General circulation model simulation of the semiannual oscillation of the tropical middle atmosphere," Journal of the Atmospheric Sciences, vol. 45, no. 21, pp. 3212-3235, 1988.

[24] L. J. Sonmor and G. P. Klaassen, "Mechanisms of gravity wave focusing in the middle atmosphere," Journal of the Atmospheric Sciences, vol. 57, no. 4, pp. 493-510, 2000.

[25] S. A. Thorpe, "Observations of parametric instability and breaking waves in an oscillating tilted tube," Journal of Fluid Mechanics, vol. 261, pp. 33-45, 1994.

[26] D. Broutman and W. R. Young, "On the interaction of smallscale oceanic internal waves with near-inertial waves," Journal of Fluid Mechanics, vol. 166, pp. 341-358, 1986.

[27] D. L. Bruhwiler and T. J. Kaper, "Wavenumber transport: scattering of small-scale internal waves by large-scale wavepackets," Journal of Fluid Mechanics, vol. 289, pp. 379-405, 1995.

[28] S. D. Eckermann, "Influence of wave propagation on the doppler spreading of atmospheric gravity waves," Journal of the Atmospheric Sciences, vol. 54, no. 21, pp. 2554-2573, 1997.

[29] J. C. Vanderhoff, J. W. Rottman, and D. Broutman, "The trapping and detrapping of short internal waves by an inertia wave," Physics of Fluids, vol. 22, no. 12, Article ID 126603, 2010.

[30] J. Vanderhoff, K. Nomura, J. Rottman, and C. Macaskill, "Doppler spreading of internal gravity waves by an inertiawave packet," Journal of Geophysical Research, vol. 113, Article ID C05018, 2008.

[31] R. L. Walterscheid, "Propagation of small-scale gravity waves through large-scale internal wave fields: Eikonal effects at lowfrequency approximation critical levels," Journal of Geophysical Research D, vol. 105, no. 14, pp. 18027-18037, 2000.

[32] R. Godoy-Diana, J. M. Chomaz, and C. Donnadieu, "Internal gravity waves in a dipolar wind: a wave-vortex interaction experiment in a stratified fluid," Journal of Fluid Mechanics, vol. 548, pp. 281-308, 2006.

[33] D. C. Fritts and T. J. Dunkerton, "A quasi-linear study of gravity wave saturation and self-acceleration," Journal of the Atmospheric Sciences, vol. 41, no. 22, pp. 3272-3289, 1984.

[34] B. R. Sutherland, "Internal wave instability: wave-wave versus wave-induced mean flow interactions," Physics of Fluids, vol. 18, no. 7, Article ID 074107, 2006.

[35] A. S. Broad, "Linear theory of momentum fluxes in 3-D flows with turning of the mean wind with height," Quarterly Journal of the Royal Meteorological Society, vol. 121, no. 528, pp. 18911902, 1995.

[36] A. S. Broad, "Do orographic gravity waves break in flows with uniform wind direction turning with height?" Quarterly Journal of the Royal Meteorological Society, vol. 125, no. 557, pp. 1695-1714, 1999.

[37] K. B. Winters and E. A. D’Asaro, “Two-dimensional instability of finite amplitude internal gravity waves packets near a critical layer," Journal of Geophysical Research, vol. 94, no. C 9, p. 12709, 1989.

[38] F. S. Henyey and N. Pomphrey, "Eikonal description of internal wave interactions: a non-diffusive picture of 'induced diffusion," Dynamics of Atmospheres and Oceans, vol. 7, no. 4, pp. 189-219, 1983.

[39] F. S. Henyey, J. Wright, and S. M. Flatté, "Energy and action ow through the internal wave field: an eikonal approach," Journal of Geophysical Research, vol. 91, pp. 8487-8495, 1986.

[40] A. Javam and L. G. Redekopp, "The transmission of spatiallycompact internal wave packets through a critical level," Dynamics of Atmospheres and Oceans, vol. 28, no. 3-4, pp. 127$138,1998$.

[41] P. Müller, G. Holloway, F. Henyey, and N. Pomphrey, "Nonlinear interactions among internal gravity waves," Reviews of Geophysics, vol. 24, pp. 493-596, 1986.

[42] K. B. Winters and E. A. D'Asaro, "Three-dimensional wave instability near a critical level," Journal of Fluid Mechanics, vol. 272, pp. 255-284, 1994.

[43] C. O. Hines, "The saturation of gravity waves in the middle atmosphere. Part II: development of Doppler-spread theory," Journal of the Atmospheric Sciences, vol. 48, no. 11, pp. 13601379, 1991.

[44] C. O. Hines, "The saturation of gravity waves in the middle atmosphere. Part IV: cutoff of the incident wave spectrum," Journal of the Atmospheric Sciences, vol. 50, no. 18, pp. 30453060, 1993.

[45] C. O. Hines, "Nonlinearity of gravity wave saturated spectra in the middle atmosphere," Geophysical Research Letters, vol. 23, no. 23, pp. 3309-3312, 1996.

[46] C. O. Hines, "Doppler-spread parameterization of gravitywave momentum deposition in the middle atmosphere. Part 1: basic formulation," Journal of Atmospheric and SolarTerrestrial Physics, vol. 59, no. 4, pp. 371-386, 1997.

[47] K. N. Sartelet, "Wave propagation inside an inertia wave. Part I: role of time dependence and scale separation," Journal of the Atmospheric Sciences, vol. 60, pp. 1433-1447, 2003.

[48] K. N. Sartelet, "Wave propagation inside an inertia wave. Part II: wave breaking," Journal of the Atmospheric Sciences, vol. 60, pp. 1448-1455, 2003.

[49] D. Broutman, "On internal wave caustics," Journal of Physical Oceanography, vol. 16, pp. 1625-1635, 1986.

[50] K. Sato, D. J. O’Sullivan, and T. J. Dunkerton, “Low-frequency inertia-gravity waves in the stratosphere revealed by threeweek continuous observation with the MU radar," Geophysical Research Letters, vol. 24, no. 14, pp. 1739-1742, 1997.

[51] R. O. R. Y. Thompson, "Observation of inertial waves in the stratosphere," Quarterly Journal of the Royal Meteorological Society, vol. 104, pp. 691-698, 1978.

[52] A. Gill, Atmosphere-Ocean Dynamics, Academic Press, 1982.

[53] W. D. Hayes, "Kinematic wave theory," Proceedings of the Royal Society of London, vol. 320, no. 1541, pp. 209-226, 1970.

[54] W. L. Jones, "Propagation of internal gravity wave in uids with shear and rotation," The Journal of Fluid Mechanics, vol. 30, pp. 439-448, 1967. 

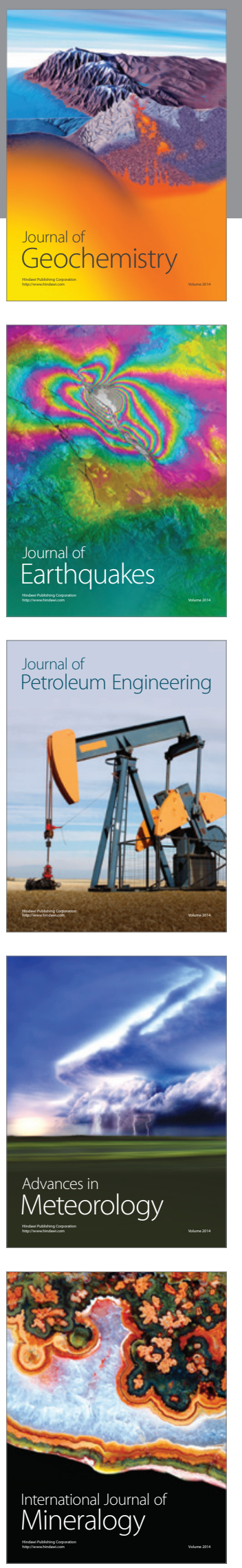
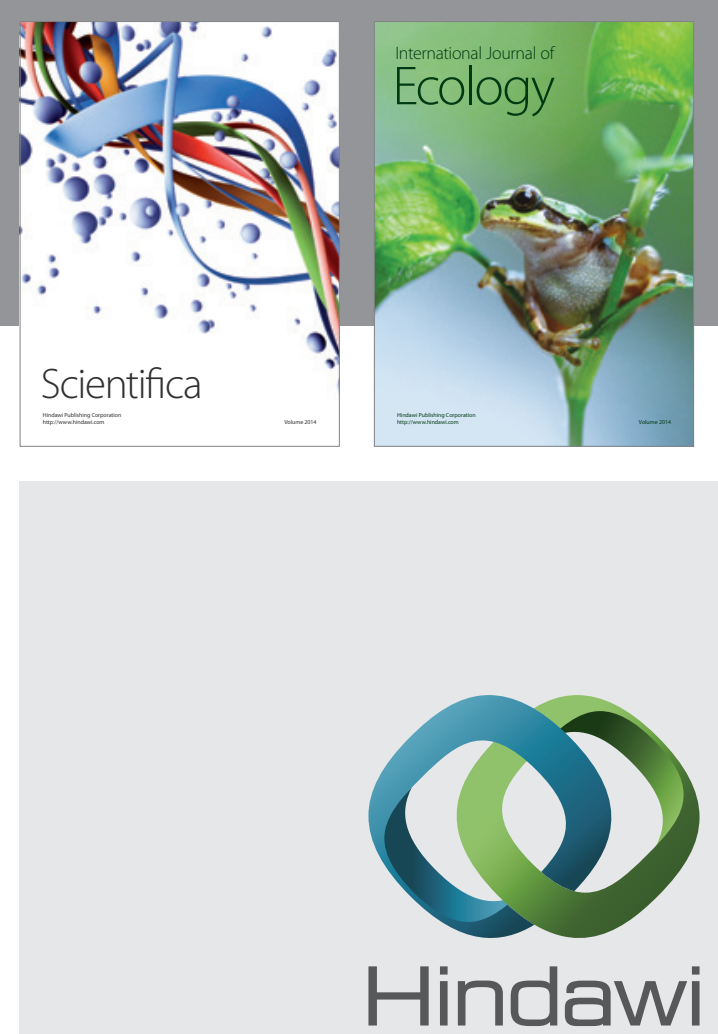

Submit your manuscripts at http://www.hindawi.com
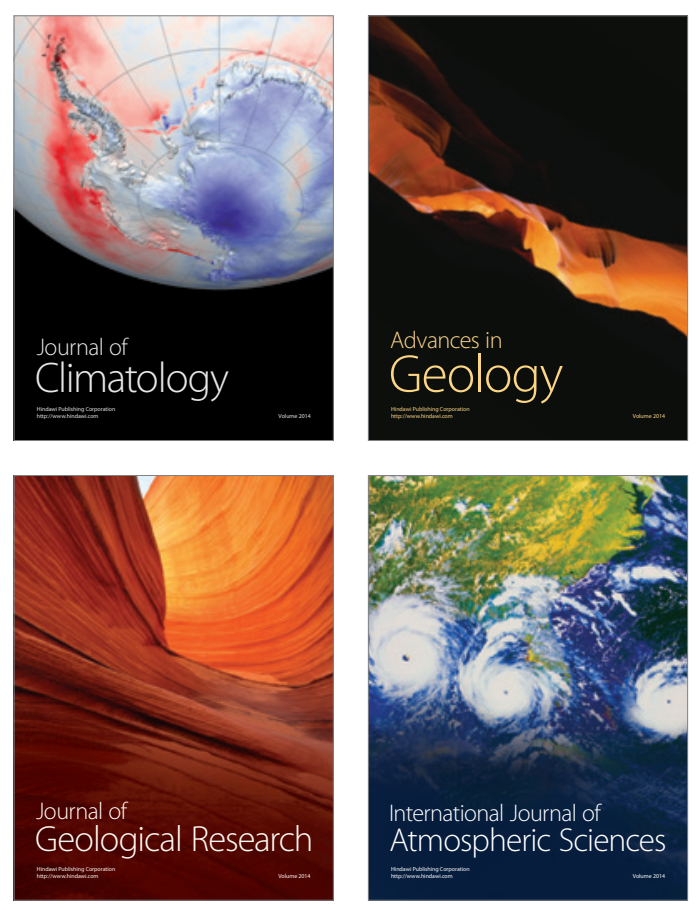
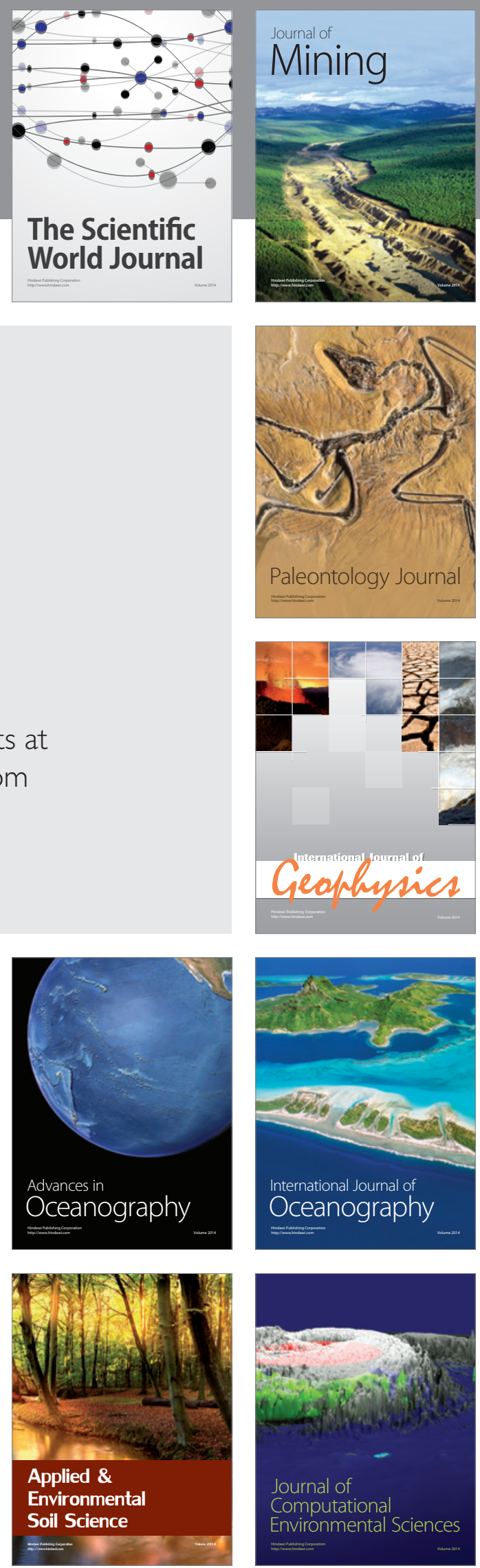